\title{
Competencias en las clases de idiomas del mundo en la virtualidad
}

\section{On-line Competency Based Learning in World Language Classes}

Aida Cristina Perdomo ${ }^{1}$
(iD Allison Tarwater Reeves ${ }^{\square}$

${ }^{1}$ Indian Land High School. Carolina del Sur, EE. UU

${ }^{2}$ Charlotte Christian School. Carolina del Norte, EE. UU

${ }^{3}$ South Mecklenburg High School. Carolina del Norte, EE. UU

\section{RESUMEN}

Los educadores del siglo XXI deberán ser valientes y arriesgarse a redireccionar los sistemas educativos hacia un desarrollo sostenible y una educación inclusiva, con un énfasis en la formación de competencias adaptables y transferibles. Además, deberán navegar entre las TIC y las necesidades de centrar el enfoque del proceso enseñanza-aprendizaje en el estudiante. Después de la pandemia del Covid-19 los profesores saben que la incertidumbre es lo único cierto y que sus herramientas más seguras son el instinto docente para encontrar la dirección que se le debe dar a sus pupilos. Este artículo investigativo está basado en una revisión documental sobre la educación por competencias, la virtualidad y la enseñanza de las lenguas del mundo basadas en la visión de UNESCO 2030. Se concluyó que la virtualidad llegó a la educación para quedarse y es importante hallar un balance TIC-personalización, porque se educa individuos que necesitan conexiones humanas fuertes y saludables. Palabras clave: Educación por competencias, TIC, virtualidad, lenguas del mundo.

\begin{abstract}
Twenty First century educators must be courageous and take risks in redirecting education systems towards sustainable development and inclusive education, all with an emphasis on the formation of adaptable and transferable skills. In addition, these professionals must navigate between ICT and the need to focus the teaching-learning process on the student. After Covid-19, teachers know that uncertainty is the only thing that is certain and that their safest tools are their own instincts to find the direction for their students. This investigative article is based on a documentary review of skills-based education through competencies, on line learning, and world language instruction based on UNESCO's 2030 vision. It was concluded that on line learning came to education to stay and it is important to recognize the needs of an ICT-personalization balance skills-based learning as we are educating individuals who need strong and healthy human connections.
\end{abstract} Keywords: Skills-based learning, ICT, on line learning, world languages. 


\section{INTRODUCCIÓN}

La educación global a partir del 2021 estará basada en competencias, tendrá componentes virtuales y deberá centrarse en las necesidades del estudiante, como ya lo venían anunciando las recomendaciones de las políticas públicas de educación de los organismos internacionales. Con el cierre obligatorio de los centros educativos ante el COVID-19, quedó demostrado que la presencialidad dejó de ser la única opción de la enseñanza-aprendizaje y se hizo evidente que sistemas educativos, profesores, educandos y padres requieren competencias tecnológicas que les permita guiar el descubrimiento del saber requerido de los ciudadanos del siglo XXI. Ante el reto que presentó la crisis sanitaria de cómo continuar la enseñanza, la reacción de emergencia fue lanzarse a la virtualidad. Comenzada la reflexión de la post pandémica el desafío es ¿cómo enseñar virtualmente y cómo demostrar el aprendizaje? Aunque la respuesta no se ha hallado, se sabe que no es universal, porque cada aula es sui generis; y está en constante evolución, porque cada día presenta nuevos retos, alternativas y esperanzas.

Mucho antes de la pandemia la UNESCO (2015) ya demandaba una reforma tendiente a la educación por competencias. En Replantear la educación: ¿hacia un bien común mundial? se analizó la problemática de la educación frente a la continua y rápida evolución de las sociedades; y, además, abrió el diálogo a un concepto de educación que preparara ciudadanos humanistas con habilidades y competencias laborales afines al entorno real. Se preguntó entonces “¿Qué instrucción se necesita para alcanzar el éxito en el siglo XXI? ¿Qué papel debe desempeñar la educación frente a la transformación de la sociedad? ¿Cuál es la organización más efectiva para el aprendizaje, dadas las circunstancias virtuales?". Las soluciones mostraron una redirección de los sistemas educativos hacia un desarrollo sostenible y permanente, y a una aproximación a la educación inclusiva en la que el profesor se convierta en el guía. Para aliviar la descoyuntara entre educación y oferta laboral, se propusieron alianzas con la industria, reforzar la responsabilidad del estado en las políticas de empleo sólidas y sobre todo un énfasis en la formación de competencias adaptables y transferibles a una carrera profesional, competencias del presente siglo y aquellas pragmáticas como son la comunicación, la alfabetización digital, la resolución de problemas, el trabajo en equipo y el espíritu de empresa" (UNESCO, 2015 p. 64).

Más adelante, la Iniciativa de Educación para el Desarrollo Sostenible del 2005-2014 de la UNESCO (2018) presentó un marco conceptual para el aprendizaje permanente a lo largo de toda la vida. Este modelo organizó el aprendizaje en los siguientes cuatro pilares:

- Aprender a conocer: el desarrollo de habilidades y conocimientos necesarios para funcionar en este mundo.

- Aprenderahacer:laadquisicióndehabilidadesaplicadasvinculadasaléxitoprofesional.

- Aprender a vivir juntos: el desarrollo de habilidades y valores sociales como el respeto y la preocupación por los demás y la apreciación de la diversidad cultural.

- Aprender a ser: el aprendizaje que contribuye a la mente, el cuerpo y el espíritu de una persona. 
Diez años después, la Organización para la Cooperación y el Desarrollo Económico (OCDE, 2015) encomendó la creación del marco conceptual al Proyecto de Definición y Selección de Competencias (DeSeCo), el cual identificó tres amplias categorías de competencias clave: 1. Es requerido que los individuos usen un gran rango de herramientas para que interactúen efectivamente con el ambiente: herramientas físicas como las TIC y socioculturales como el lenguaje; 2. La interdependencia del mundo hace necesario que las personas se comuniquen con otras, y debido a la diversidad humana, es indispensable poder actuar interculturalmente; 3. Es necesario que los individuos se responsabilicen de manejar sus vidas, situándose en un contexto social amplio y proceder de manera independiente con responsabilidad social (OCDE, 2015).

A la misma vez, la OCDE elaboró un marco curricular que contempla ocho competencias básicas de enseñanza: tratamiento de la información y competencia digital (TIC), competencia en comunicación lingüística, competencia en el conocimiento y la interacción con el mundo físico, competencia para aprender a aprender (para la UNESCO aprender a conocer), competencia autonomía e iniciativa personal, social y ciudadana; competencia cultural y artística; y competencia matemática.

Por su parte, la Unión Europea, un referente importante por cuanto unifica la educación en 27 países independientes, entiende las competencias como "la capacidad de responder a demandas complejas y llevar a cabo tareas diversas de forma adecuada" (Galván Estrada, 2010). Y agrega que para lograr una respuesta eficaz se requiere la mezcla de componentes sociales, emocionales y físicos. El informe DeSeCo anuncia que las competen- cias nacen de la combinación del saber, del hacer, de la actitud y motivación del individuo que decide completar una tarea eficazmente. "Por lo tanto una competencia clave aúna la parte teórica con la práctica y las motivaciones internas que la impulsan en aras de alcanzar el éxito de la acción" (OCDE, 2015).

Este artículo se enfoca en cuatro competencias que nos atañen como profesoras de español en Carolina del Norte, Estados Unidos, entendiendo que las TIC están en cada una de ellas: las competencias para aprender a aprender, comunicación lingüística, social y ciudadana; y la cultural y artística.

\section{Competencia: Aprender a aprender en la modalidad virtual}

El impacto que ejercen la bioelectrónica, el internet, la producción de software y la comunicación digital en el proceso de transmisión del conocimiento es innegable e imparable. La modalidad virtual cada vez cobra más relevancia porque la idea tradicional del conocimiento adquirió otra definición. Hoy la prioridad no es el adquirir y retener el conocimiento, sino saber dónde y cómo encontrarlo para usarlo en el momento en que se necesite; la rapidez con que se accede al saber que permita realizar una función o resuelva una problemática. Estos conceptos y escenarios novedosos suponen una adaptación del sistema educativo para preparar individuos capaces de relacionarse con un entorno altamente digitalizado. Se demandan instituciones y docentes versados en tecnología didáctica, en plataformas inteligentes, currículo interactivo y bibliotecas digitales.

La modalidad virtual del aprendizaje o las plataformas inteligentes requieren competencias tecnológicas que permita guiar el 
descubrimiento del saber requerido de los ciudadanos del siglo XXI y son la respuesta a las demandas de los estudiantes de la década del 2020 que pertenecen a la Generación Z, nacida entre 1994 y 2010, o sea, los estudiantes que encontramos en nuestras clases de español de bachillerato y que han crecido con todo lo que la internet les ofrece. Según Carla Torreiro Rondón (2021), profesora y fundadora de Munus Lingua en España, las plataformas inteligentes son una tendencia universal $\mathrm{y}$ ofrecen ventajas para educando y docentes.

Para los estudiantes:

- Aportan flexibilidad a la forma de aprender y la organización de los recursos

- Emiten estadísticas y resultados sobre la forma en la que los alumnos usan las plataformas con el objeto de su posterior facilitación y mejora

- Reiteran los aprendizajes de conceptos y procedimientos con alternativas de recursos y secuencias de aprendizaje hasta conseguir las competencias necesarias

- Proporcionan una retroalimentación personalizada, con base en datos, para poder corregir errores y aprender de la experiencia.

Para los docentes:

- Facilitan la toma de decisiones en cuanto a planificación, orientación o apoyo a los alumnos

- Ofrecen una base de datos relevante para mejorar el aprendizaje y así sepan exactamente cómo mejorar tanto profesores como alumnos.

En los experimentos de los últimos años se han creado modelos tendientes a guiar la implementación pertinente a la tecnología en las aulas, los de mayor relevancia en la virtua- lidad han sido, el modelo SAMR y la rueda de la taxonomía. El primero, SMAR (Sustitución, Aumentación, Modificación, y Redefinición) guía el diseño innovador y justificado de la tecnología; el segundo, la rueda de la taxonomía es una adaptación moderna de la Taxonomía de Bloom que data de 1956 y explica cómo incluir las plataformas inteligentes en el currículo contemporáneo. Su creador, Paul Hopkin (Puentedura, 2015), también elaboró una lista de las habilidades que los empleadores buscan en el graduado de educación superior en el Siglo XXI. Entre ellas se encuentran: conocer y dominar la tecnología relevante a su profesión; demostrar pasión y entusiasmo por su carrera; demostrar empatía y capacidad de colaboración aceptando las debilidades propias y de los demás; saber negociar con transparencia y demostrando respeto por las opiniones del otro; ser perseverante, positivo y mantener la calma en momentos de alto estrés; manejar y organizar el tiempo con eficiencia y poder efectuar presentaciones con alta oratoria.

Es necesario reconocer que el currículo contemporáneo debe ofrecer variedad de plataformas teniendo en cuenta la realidad del entorno del estudiante. La brecha digital, rompe con los principios de equidad en la educación, ya que los estudiantes de sectores vulnerables solo podrían tener acceso en ambientes comunitarios, como las bibliotecas, los centros culturales en donde el tiempo del uso personalizado es limitado.

El currículo de las plataformas inteligentes producirá un graduado del Siglo XXI interesado en aumentar su bagaje académico durante toda su vida, que use la tecnología para acceder al conocimiento relevante a su campo 
profesional y su entorno; y que posea la habilidad para entender y emplear las TIC a medida que avanzan y evolucionan. El currículo en plataformas inteligentes es parte integral en la construcción de los cuatro pilares sobre los cuales se debe levantar el currículo del Siglo XXI.

\section{Competencia en comunicación lingüística en la modalidad virtual}

En el preámbulo de La Ley Orgánica de Educación (LOE) que regula la educación de la Unión Europea, se dejó claro que uno de los objetivos más importantes del sistema educativo es la competencia en comunicación lingüística, definida como capacidad de comunicar y comprender oral y textualmente ideas, acontecimientos y reacciones; además hacerlo atendiendo los códigos y registros lingüísticos según el entorno en el que se encuentre. (Barbero et al, 2008). Esta noción ha calado en los sistemas educativos de España. Por ejemplo, en Cantabria el currículo dispone "la utilización del lenguaje como instrumento de comunicación oral y escrita, de representación, interpretación y comprensión de la realidad, de construcción y comunicación del conocimiento y de organización y autorregulación del pensamiento, las emociones y la conducta" (Barbero et al., 2008, p. 7). La competencia en comunicación lingüística incluye habilidades en la lengua materna y una segunda lengua del mundo (lengua extranjera). Esta última desarrolla capacidad de empatía y comprensión sobre la cultura del idioma extranjero y con ello se da la multiculturalidad, objetivo final de la convivencia con el otro.

Se evidencia la competencia de comunicación lingüística cuando un individuo puede utilizar la lengua para comunicar espontáneamente ideas y sentimientos, demostrando conocimiento de las convenciones sociales, elementos culturales y el registro lingüístico de su interlocutor. Como profesoras de idiomas del mundo, sabemos que adquirir las habilidades lingüísticas en una segunda lengua requiere entender cómo funciona el idioma; y cómo y cuándo poderlo usar obedeciendo los códigos apropiados a cada escenario; pero reconocemos que es primordial que los aprendices tengan contenido y sepan de qué hablar. Se produce la competencia de comunicación lingüística cuando el individuo ha desarrollado curiosidad e interés en los asuntos culturales y siente que su voz es bienvenida en un entorno seguro y cálido; cuando se siente confiado y respetado para probar, equivocarse, autocorregirse y volver a intentarlo. El educador como tutor, estimulador, conductor y guía debe adaptar el currículo oficialmente mandado a tareas que incluyan virtualidad y mini lecciones conductoras al aprendizaje. Maldonado (2002) resalta que la competencia lingüística es inherente al ser humano y recomienda variedades de juegos y roles que lo exponga a interacciones con otras personas, reflexione sobre su postura y negocie con los otros. Aprovechando las TIC se puede concertar un campeonato internacional de bailes típicos latinoamericanos entre estudiantes de español en EE. UU. y Bolivia, por ejemplo. O un concurso de cuentos cortos bilingües entre estudiantes de español e inglés como segundas lenguas.

\section{Competencia social y ciudadana en la mo- dalidad virtual}

De acuerdo con Pagès la competencia social y ciudadana (CSC) es "fundamental en el desarrollo del proyecto educativo, pues los centros y sus aulas deben convertirse en espacios fundamentales para el crecimiento personal del alumnado y para el aprendizaje de la 
democracia y, en consecuencia, para su desarrollo y su aplicación" (2009). La CSC es una de las competencias básicas para el siglo XXI y supone distinguir la realidad de la sociedad, afrontar las relaciones y las desavenencias con ética, con integridad personal y respetando siempre los principios democráticos; $\mathrm{y}$, además, estar comprometido con la convivencia en armonía, el bienestar común y el respeto de los deberes y derechos cívicos del individuo (Pereira, 2007, p.25).

La CSC se logra cuando el educando conoce y entiende las bases sobre los cuales descansa una sociedad democrática, haciendo una reflexión crítica de las ideas de: soberanía de la mayoría, libertad, apoyo a las causas nobles del otro, responsabilidad compartida, participación, acatamiento a los derechos y deberes promovidos por los organismos internacionales (Monzonís M. \& Capllonch, 2015).

La adquisición de la CSC es un proceso continuo en el que los estudiantes, experimentando la cooperación, la convivencia, la participación y la toma de decisiones, aprenden lo que significa operar en una sociedad democrática y el compromiso que tienen de mejorarla. Uno de los métodos que se utilizan en las clases de idiomas para lograr el cumplimiento de competencias es el aprendizaje basado en proyectos (ABP). A través de proyectos los estudiantes podrán investigar y crear conocimiento sobre la pobreza, la desigualdad, los conflictos bélicos, la diversidad de etnias, los problemas ecológicos; realidades que también se viven en EE. UU. (Rivero Sánchez, 2016). Una de las grandes ventajas del ABP es la facilidad como puede pasar de la enseñanza presencial a la híbrida o virtual, gracias a las plataformas que permiten la colaboración. El ABP no solo permite el cono- cer las realidades sociales, sino que también permite que los estudiantes aprendan a convivir con los demás, puesto que, en la ejecución del proyecto, los estudiantes tendrán que escuchar, negociar, ceder, aceptar recomendaciones, y sobretodo desarrollar tolerancia hacia los demás integrantes del grupo (Monzonís Martínez \& Capllonch Bujosa, 2015).

\section{Competencia cultural y artística en la mo- dalidad virtual}

La competencia cultural y artística consiste en reconocer las manifestaciones culturales y artísticas de los pueblos, comprender el origen y su intención, apreciar el mensaje y respetarlo es parte de las habilidades que todos los individuos deben desarrollar para su enriquecimiento personal y su participación en la protección del patrimonio cultural universal. De igual forma, esta competencia incluye el conocer básicamente las principales presentaciones, reglas y variantes de los múltiples modelos artísticos, y poder utilizar tales conocimientos para expresar ideas propias de manera única y creativa (Buela Fresno, 2017). El significado de la competencia cultural y artística para el estudiante, se demuestra en el interés por el mundo de las bellas artes, que son un reflejo de la identidad y afiliación con alguna cultura, pero que al mismo tiempo respeta y admira las producciones artísticas del otro, con quien buscará una conexión artística-cultural genuina, ya sea pasiva o activa. En las aulas de idiomas del mundo es bien conocido que enseñar un lenguaje es exponer su cultura, y por ello se incluye educación artística como música, teatro, artes visuales, literatura y danza. Los alumnos pueden elaborar una biografía de audio-lectura de Picasso y Kahlo; liderar una visita virtual al Museo de Botero; invitar a un seminario con expertos en tatuajes y arte callejero, en 
Tegucigalpa. Ya afirma la sabiduría popular que una imagen vale más que mil palabras y la virtualidad nos permite tener las imágenes al alcance de los pulgares. Podemos mostrar el interior del Museo del Prado, las pirámides de Teotihuacán, la población garífuna y hasta lo profundo de la catedral de la mina de sal en Zipaquirá, desde nuestro salón de clases.

Es importante reconocer que vivimos en una sociedad con múltiples influencias estéticas que contribuyen en la construcción de identidad y que influyen en las ideas que construimos sobre el mundo. El desarrollo de las TIC ha producido un consumo masivo de música $\mathrm{e}$ imágenes y con ello el contexto cultural presenta notables transformaciones. Hoy gozamos de expresiones artísticas diversas: música, danza, artes visuales y artes escénicas que pueden ser utilizadas en el aula de idiomas del mundo a diario, ya sea a través de la canción de moda que se les pega a todos como ocurrió con Despacito, con los poemas cantados de José Martí como Guantanamera, o con los cuentos cortos del realismo mágico de García Márquez.

\section{CONCLUSIONES}

Las autoras, como profesoras de español en EE. UU. con altos rendimientos académicos y décadas de experiencia, reconocemos que enseñar por competencia en vez de calificaciones requiere un cambio conceptual, y por ende una adaptación curricular, de tareas y evaluaciones. Hemos vivido la transición de la enseñanza tradicional a la enseñanza por competencias y reconocemos que amerita el esfuerzo porque garantiza el mejoramiento de la experiencia enseñanza-aprendizaje, y también, la formación del ciudadano del siglo XXI.

Los educadores del Siglo XXI necesitan ser atrevidos y valientes para probar e improvisar apoyándose en las experiencias previas. Más que nunca, los profesores deben aceptar que la incertidumbre es lo único cierto. Deben lanzarse a usar las herramientas que posee para abrirse camino, y, en la aventura, crear nuevos instrumentos que se adapten a lo nunca antes imaginado. Además, confiar en el instinto docente para encontrar la dirección que se le debe dar a sus pupilos.

La virtualidad llegó a la educación para quedarse y es importante reconocer el menester de un balance TIC-personalización, porque estamos educando individuos que necesitan conexiones humanas fuertes y saludables. El uso de competencias en la modalidad virtual exige mayor autodisciplina y autodeterminación de los estudiantes. Y los profesores deben fijar objetivos claramente definidos para que la tecnología no distraiga del propósito de la tarea.

Las TIC utilizadas efectivamente en el aula formarán los individuos adaptables, independientes y racionales que exige el siglo XXI, aquellos que la modalidad presencial tradicional no produjo porque la institución, el profesor sabio y el currículo rígido no permitieron pensar en las necesidades del estudiante y su entorno. La tecnología, como herramienta novedosa y regeneradora, supone agilidad, soltura, creatividad, soluciones múltiples y colaboración. Para ello es indispensable preparar al docente en el uso efectivo de las TIC.

Las clases de idiomas del mundo son el espacio perfecto para el aprendizaje de competencias, los currículos interdisciplinarios permiten elaborar tareas y asignaciones basadas en habilidades y destrezas que demuestran el uso de la lengua. Esta combinación de saberes, habilidades y actitudes permite al estu 
diante interactuar con su entorno de Así se da cumplimiento a las competencias de manera interpersonal, presentacio- la UNESCO aprender a ser, aprender a hacer, nal e interpretativa mostrando una com- aprender a vivir juntos y aprender a conocer. petencia intercultural y lingüística.

\section{REFERENCIAS BIBLIOGRÁFICAS}

Barbero, J., Maestro, A., Pitcairn, C., \& Saiz, A. (2008). Las competencias básicas en el área de Lenguas Extranjeras. Cuadernos de educación de Cantabria, (6). https://www.educantabria.es/docs/info_institucional/publicaciones/2008/Cuadernos Educacion_6.pdf?phpMyAdmin=DxoCAdBlc\%2CANuNIkvc-WZcMiFvc

Buela Fresno, I. (2017). El componente cultural en la enseñanza/aprendizaje de lenguas extranjeras. Uvadoc.uva.es. http://uvadoc.uva.es/handle/10324/27693

Galván Estrada, N. G. (2010). Educar en competencias. DOCERE, 2, 27-30. https://doi.org/10.33064/2010docere22729

González, S., Catalán, M. \& Lara, J. M. (2011). La competencia social y ciudadana en Educación Física. En O. R. Contreras \& R. Cuevas (Eds.), Las competencias básicas desde Educación Física (pp. 63-77). Barcelona: INDE.

Maldonado M.A. (2002). Las Competencias una Opción de Vida. Bogotá: Ecoe. https://bit.ly/3eVbsdu

Monzonís Martínez, N., \& Capllonch Bujosa, M. (2015). Mejorar la competencia social y ciudadana: innovación desde educación física y tutoría. Retos, 28, 256-262 https://www.redalyc.org/pdf/3457/345741428045.pdf

Organización para la Cooperación y el Desarrollo Económico (OCDE). (2015). LA DEFINICIÓN Y SELECCIÓN DE COMPETENCIAS CLAVE Resumen ejecutivo. https://www.deseco.ch/bfs/deseco/en/index/03/02.parsys.78532.downloadList.94248. DownloadFile.tmp/2005.dscexecutivesummary.sp.pdf

Pagès, J. (2009). Competencia social y ciudadana. Core.ac.uk, 187, 7-11. https://core.ac.uk/reader/132092910

Pereira, M. M. V. (2007). Desarrollo Curricular de la Loe: Las Competencias Básicas Y $\mathrm{Su}$ Tratamiento en la Educación Física en Primaria. In Google Books. Lulu.com. https://www.google.com/books/edition/Desarrollo_Curricular_de_la_Loe_Las_Comp/ WfKRV2qoNEMC?hl=en\&gbpv=1\&dq=Comprender + la + realidad + social + en + que + se +vive 
Puentedura, R. R. (2015). SAMR: A Brief Introduction. https://bit.ly/3kRNELx

Rivero Sánchez, L. (2016). Universidad Internacional de La Rioja Facultad de Educación. https://reunir.unir.net/bitstream/handle/123456789/3580/RIVEIRO\%20SANCHEZ\%2C\%20LIDIA.pdf?sequence $=1 \&$ isAllowed $=\mathrm{y}$

Torriero Rondón, C. (2021). 5 tendencias de educación para el 2021. mba.americaeconomia. com; América Economía. https://bit.ly/3eU3dhO

UNESCO. (2015). Replantear la educación: ¿Hacia un bien común mundial? UNESCO. https://bit.ly/3jlbleh

UNESCO. (2018, September 3). UN Decade of ESD. UNESCO. https://bit.ly/36WJCJH 